\title{
Effectiveness of interventions for adults with mild to moderate intellectual disabilities and mental health problems: systematic review and meta-analysis
}

\author{
Nadine Koslowski, * Kristina Klein, * Katrin Arnold, Markus Kösters, Matthias Schützwohl, \\ Hans Joachim Salize and Bernd Puschner
}

\section{Background}

There is a lack of available evidence in relation to the effectiveness of interventions for adults with mild to moderate intellectual disability and mental health problems.

\begin{abstract}
Aims
To evaluate the efficacy of interventions for adults with mild to moderate intellectual disabilities and co-occurring mental health problems.

\section{Method}

An electronic literature search of the databases Medline, EMBASE, PSycINFO and EBM Reviews aimed at identifying randomised controlled trials (RCTs) and controlled trials testing any type of intervention (psychotherapy, biological or system level) for people with mild to moderate intellectual disabilities (IQ score 35-69) targeting comorbid mental health problems. Additionally a meta-analysis was conducted.
\end{abstract}

\section{Results}

Twelve studies met the inclusion criteria. No significant effect was found for the predefined outcome domains behavioural problems, depression, anxiety, quality of life and functioning. The effect size for depression $(d=0.49)$ was moderate but non-significant. Quality of studies was moderate and heterogeneity was high.

\section{Conclusions}

There is no compelling evidence supporting interventions aiming at improving mental health problems in people with mild to moderate intellectual disability. The number of available trials is too low for definite conclusions. Some interventions are promising and should be evaluated further in larger and more rigorous trials.

\section{Declaration of interest}

None.

\section{Copyright and usage}

(c) The Royal College of Psychiatrists 2016.
Prevalence of mental illness is notable in people with intellectual disabilities. $^{1-4}$ Mental health problems have a negative impact on the lives of many people with such disabilities, ${ }^{5}$ and evidence-based interventions tailored to the needs of this vulnerable population are called for. Over past years some reviews have summarised the evidence for a wide range of interventions including psychotherapy and biological and system-level interventions for people with intellectual disabilities and comorbid mental illness. Drawing upon uncontrolled studies or case reports, reviews showed that behavioural interventions - especially cognitive-behavioural therapy (CBT) - are effective in reducing depression, anxiety, anger and challenging behaviour in people with intellectual disabilities. ${ }^{6,7}$ Furthermore, challenging behaviour has been found to be hardly affected by massage therapy. ${ }^{8}$ There is also limited evidence for the use of antipsychotic medication such as risperidone to reduce behaviour problems in people with intellectual disabilities, which also bear a high risk of serious adverse effects. ${ }^{9-11}$

Available evidence from controlled studies is scarce when investigating specific interventions or combinations of interventions. For example, a meta-analysis showed rather large effect sizes for anger management in people with intellectual disabilities, albeit based upon only two randomised controlled trials (RCTs) and six non-randomised controlled trials. ${ }^{12}$ Based on six RCTs, a Cochrane review showed that system-level interventions did not produce significant effects on functioning or quality of life of people with intellectual disabilities and mental health problems. ${ }^{13}$ However, some meta-analyses using a broader approach including the investigation of a wider range of interventions have been put forth. For example, Heyvaert et al, on the basis of 30 controlled

*Joint first authors. studies, reported an overall large effect $(d=0.671)$ for interventions targeting challenging behaviour in people with intellectual disabilities, ${ }^{14}$ which varied by type of intervention (psychotherapy $d=0.752$, biological $d=0.646$, system-level $d=0.624) .{ }^{14}$ Furthermore, on the basis of 14 trials, Vereenooghe \& Langdon also found a large effect $(g=0.682)$ for psychotherapy in people with intellectual disabilities, with subgroup analyses showing somewhat higher effect sizes for CBT. ${ }^{15}$

Taken together, the majority of reviews summarising the evidence on interventions targeting mental health problems in people with intellectual disabilities did not employ a systematic search strategy, did not rely on controlled trials, or included studies with children and adolescents, making questionable the transfer of results to adults. The few meta-analyses reported overall moderate to large effect sizes. Thus, findings should be interpreted with caution as the number of rigorously controlled studies in the field is low, as are sample sizes of included studies, and outcome measures are heterogeneous. Furthermore, there is solid evidence that the manifestations of mental illness and their appropriate treatment options, especially in the case of interventions requiring a fair amount of language skills such as psychotherapy, differ substantially by level of severity of intellectual disabilities. ${ }^{6,16}$ So far, none of the systematic reviews, except for one, ${ }^{7}$ was tailored to identify evidence for people with a defined severity level of intellectual disabilities (mild, moderate, severe or profound). Findings from the existing reviews, which were not specifically based on studies examining adults with mild to moderate intellectual disabilities, may not be valid for this subgroup. We therefore systematically reviewed the evidence from controlled studies examining the effectiveness of diverse interventions (psychotherapy, biological and system-level approaches) for people with mild to 
moderate intellectual disabilities and comorbid mental health problems.

\section{Method}

The systematic review was performed in accordance with the Preferred Reporting Items for Systematic Reviews and Meta-Analyses (PRISMA) checklist. ${ }^{17}$

\section{Inclusion and exclusion criteria}

We included English-language articles published after 1980 reporting the results of randomised controlled trials or controlled studies using parallel group designs to test any type of intervention focusing on the treatment of mental health problems for adults with intellectual disability and comorbid mental illness or behavioural problems. Study participants had to be identified as intellectually disabled with any comorbid mental illness or any behaviour problems. At least $75 \%$ of the sample had to be aged 18-64 years and had to function in the range of mild to moderate intellectual disabilities defined by an IQ score of 35-69 or author classification.

We did not include studies in which the intellectual disability levels of participants were not reported, or only an average IQ score was presented without information on the range of IQ scores or the distribution of participants over severity levels of intellectual disability. Studies reporting only scores on the British Picture Vocabulary Scale were excluded as this is a measure of receptive language abilities and thus limited to the verbal component of intelligence. ${ }^{18,19}$ Studies in which more than $25 \%$ of the study participants were outside the designated age range were also excluded. Furthermore, studies testing interventions that aimed at the primary prevention of mental illness or behavioural problems were excluded.

\section{Search strategy}

The electronic databases Medline, EMBASE, PsycINFO and EBM Reviews were searched combining key terms describing indications, interventions and study types (see online Table DS1). The electronic search was run in April 2013 and updated in September 2014. References of included studies and related reviews were crosschecked for further relevant literature. We performed a hand search for further relevant literature in the American Journal of Mental Retardation, Journal of Applied Research in Intellectual Disabilities and Journal of Mental Health Research in Intellectual Disabilities. In cases of missing or unclear data we contacted the authors for further information.

\section{Study selection}

Each study was independently inspected by two of three reviewers (K.K., K.A. and N.K.). Any disagreements between the reviewers were resolved by discussion.

\section{Data extraction and quality assessment}

Variables extracted from primary data were sample size and patient characteristics (gender, age and diagnosis), type of intervention, length of follow-up, design and outcome measures. We extracted any clinical outcomes related to mental health (e.g. symptom severity, psychopathology) and any social outcomes (e.g. quality of life, social functioning) reported in the primary studies. Outcomes were summarised in the following domains: behavioural problems, depressive symptoms, anxiety symptoms, quality of life and functioning. We assessed the quality of the included studies by means of the Cochrane risk of bias tool which consists of seven domains: sequence generation, allocation concealment, masking (blinding) of participants and personnel, masking of outcome assessment, incomplete outcome data, selective outcome reporting and 'other issues. ${ }^{20}$ Each domain and overall risk of bias was assessed and categorised as low, high or unclear.

\section{Data aggregation}

Data synthesis implied both a qualitative analysis to provide a narrative overview and quantitative analyses (meta-analyses) for the five outcome domains which were carried out separately for all trials with sufficient data (mean, standard deviation, number of participants). If studies reported more than one measure in each domain, we calculated a single mean effect for each study. Outcome domains included the following measures:

(a) behavioural problems: Checklist of Challenging Behaviour, Stereotypic Self-Stimulating Behavior, the Behavior Checklist, the Aberrant Behavior Checklist, the Modified Overt Aggression Scale, the Provocation Index and the Anger Inventory;

(b) depressive symptoms: Beck Depression Inventory and the Automatic Thoughts Questionnaire - Revised;

(c) anxiety symptoms: Beck Anxiety Inventory and the Glasgow Anxiety Scale;

(d) quality of life: Quality of Life Questionnaire, Manchester Short Assessment of Quality of Life, World Health Organization Quality of Life-Bref and the Comprehensive Quality of Life Scale - Intellectual Disability;

(e) functioning: Global Assessment of Functioning, and the American Association for Mental Deficiency Adaptive Behavior Scale.

The direction of effect was standardised with positive values indicating a better outcome in the intervention group. As studies were expected to differ in duration of follow-up, we selected the end-point of the intervention for meta-analysis. Effect sizes and their $95 \%$ confidence intervals were calculated as standardised mean differences (SMDs). In case of missing standard deviations, these were calculated (e.g. from standard error) or imputed (from pre-intervention scores) if possible. Effect sizes were interpreted according Cohen's rule of thumb (small 0.20-0.49, medium $0.50-0.79$, large 0.8 and higher).$^{21}$ The meta-analysis was performed using a random effects model. We calculated $I^{2}$ scores to examine heterogeneity between studies. ${ }^{20}$ The results of the meta-analysis were presented as forest plots.

\section{Results}

The electronic search identified 4602 publications (Fig. 1). Two hand-search runs yielded another 110 potentially relevant articles. After removal of duplicates 3072 studies remained. The initial title and abstract screening identified 146 potentially relevant studies which were screened in full text. Of these, 12 trials fulfilled the inclusion criteria. The main reasons for exclusion were that studies were not controlled or did not report information about the participants' severity of intellectual disability.

\section{Characteristics of included studies}

The included studies were published between 1980 and 2014 . Eleven were RCTs, ${ }^{16,22-31}$ and one was a non-randomised study. ${ }^{32}$ Study characteristics are summarised online in Table DS2. Ten of 


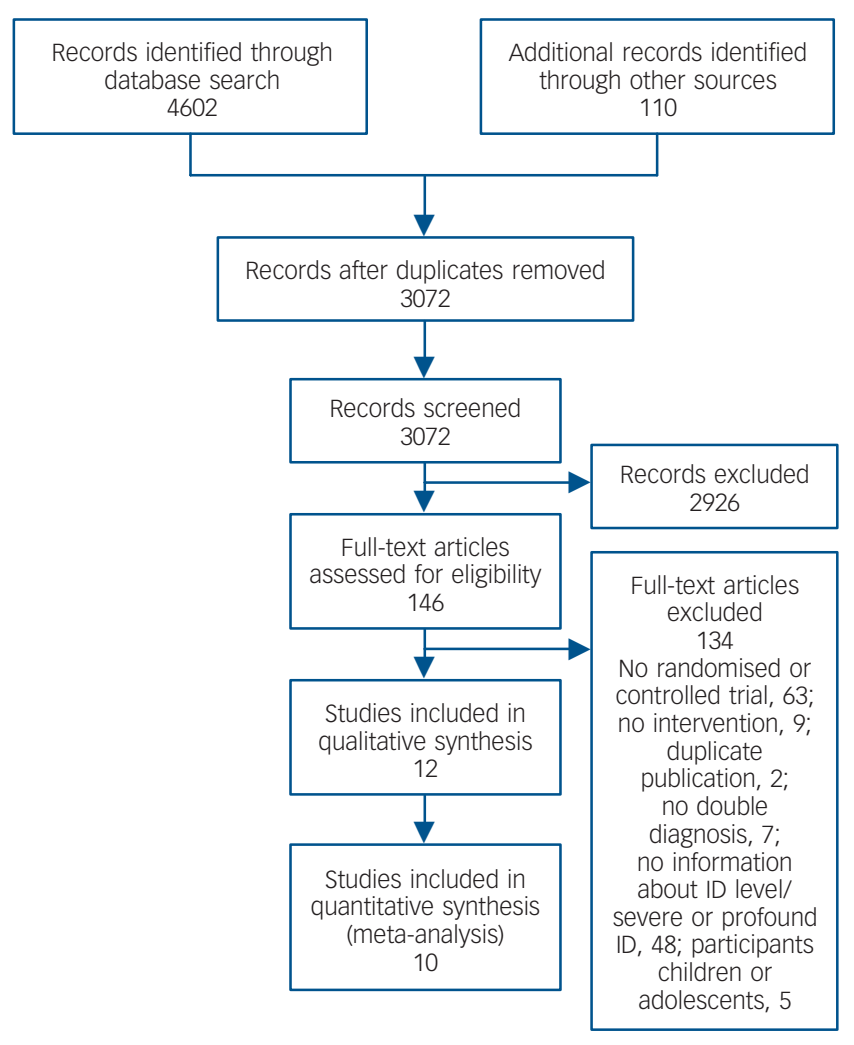

Fig. 1 Selection of studies.

the twelve included studies were conducted in a single country: USA, ${ }^{16,32}$ UK, ${ }^{24,25,28,30,31}$ Australia, ${ }^{26,27}$ and China. ${ }^{22}$ Six studies were multicentre trials, ${ }^{23-25,28-30}$ two of which were multinational - one conducted in South Africa, UK, Canada and Belgium, ${ }^{23}$ and the other in the UK and Australia. ${ }^{29}$ Studies were implemented in community, ${ }^{28,29}$ supported employment, ${ }^{26,27,32}$ or special service settings. ${ }^{16,22,24,25,30,31}$ One study did not report the setting. ${ }^{23}$

The mean sample size was 60.3 (s.d. $=44.6$; median 48 , range 14-179), with larger sample sizes in biological studies. On average $44 \%$ (s.d. $=12$; median 40 ) of study participants were female. The majority of study participants were adults. In two studies some participants were less than 18 years old, ${ }^{22,32,}$ and in four studies a few participants were over 65 years old (maximum 70 years). ${ }^{16,22,24,28}$ Participants' mental health problems were described as mental disorders or behavioural problems diagnosed according to DSM or ICD-10 criteria in four studies, ${ }^{16,23-25}$ as depressive symptoms in three studies, ${ }^{26-28}$ and as behaviour problems including aggressive or inappropriate behaviour or problems handling anger in five studies. ${ }^{22,29-32}$ Interventions delivered were classified as biological (risperidone, risperidone and haloperidol, and multisensory therapy) in three studies, ${ }^{22,23,29}$ system-level (assertive community treatment and case management) in three studies, ${ }^{16,25,28}$ and as psychotherapy (CBT) in six studies. ${ }^{24,26,27,30-32}$ Treatment outcome domains examined were behavioural problems, ${ }^{16,22,23,25,29-32}$ depressive symptoms, ${ }^{24,26,27,30}$ anxiety symptoms, ${ }^{24,30}$ quality of life, ${ }^{25,28-30}$ and functioning. ${ }^{16,25,28}$ Control conditions were active treatment, ${ }^{16,22,25,28}$ waiting list, ${ }^{26,27,30,31}$ or treatment as usual. ${ }^{23,24,26,27,30,31}$ Furthermore, one study compared anger management with three control conditions, ${ }^{32}$ one placebocontrolled study compared risperidone and haloperidol, ${ }^{29}$ and one study compared risperidone with placebo. ${ }^{23}$ Most of the included studies measured the impact of the intervention at the end of treatment or within 3 weeks thereafter. One study assessed the impact of the intervention 6 months after initiation of treatment. ${ }^{25}$ All included studies, except for three, ${ }^{23,25,28}$ evaluated long-term effects. Length of follow-up ranged between 5 weeks ${ }^{22,32}$ and 2 years. ${ }^{16}$

\section{Risk of bias}

The overall methodological quality of the included studies was modest (Fig. 2). With one exception, ${ }^{29}$ studies had unclear risk in at least one domain. Furthermore, we judged four studies as having high risk in at least one domain (see online Table DS3). ${ }^{22,25,26,32}$

\section{Effects on outcome domains}

Of the twelve included studies, two were excluded from quantitative synthesis because of insufficient data or data being markedly skewed, ${ }^{32,29}$ resulting in ten studies subjected to meta-analysis.

\section{Random sequence generation (selection bias) \\ Allocation concealment (selection bias) \\ Masking of participants and personnel (performance bias)}

Masking of outcome assessment (detection bias)

Incomplete outcome data (attrition bias)

Selective reporting (reporting bias)

Low risk of bias

Unclear risk of bias

Other bias

High risk of bias

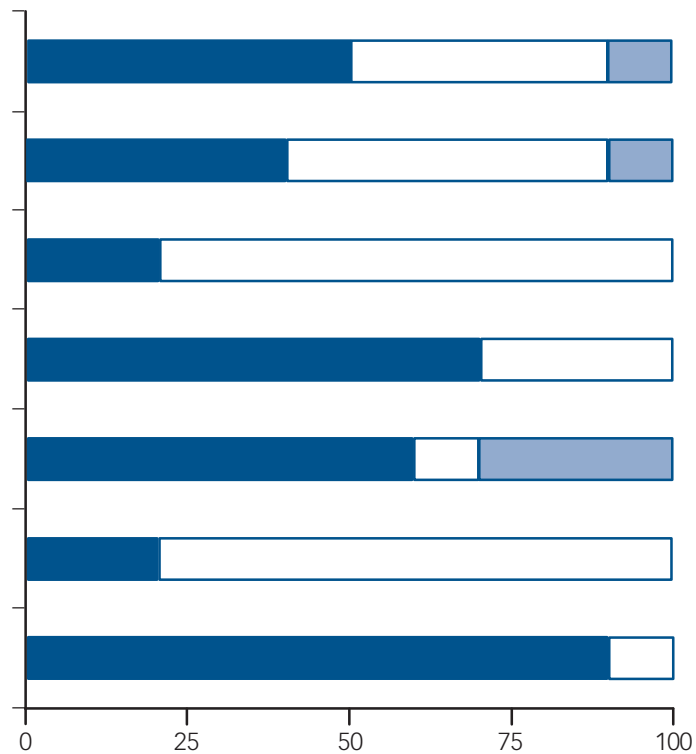




\section{Behavioural problems}

Six studies (total $n=402$ ) showed no overall effect ( $d=0.03$, 95\% CI -0.77 to $0.84 ; P=0.94$ ) (Fig. 3 ). ${ }^{16,22,23,25,30,31}$ Interventions delivered were psychotherapy, ${ }^{30,31}$ biological treatment, ${ }^{22,23}$ and system-level approaches, ${ }^{16,25}$ in two studies each. Heterogeneity was high $\left(I^{2}=92 \%\right)$. A large and statistically significant effect in favour of the control condition was found in one study. ${ }^{22}$ All other studies included showed positive effect sizes, although only one study showed a statistically significant effect. ${ }^{22}$

Depressive symptoms

Four studies, all of which tested psychotherapy (total $n=283$ ), showed a moderate effect size which was non-significant $(d=0.49,95 \%$ CI -0.05 to $1.03 ; P=0.08) .{ }^{24,26,27,30}$ Heterogeneity between studies was high $\left(I^{2}=74 \% ; P=0.009\right)$ (Fig. 3).

\section{Anxiety symptoms}

Two studies testing psychotherapy (total $n=185$ ) yielded no significant effect $(d=0.15,95 \%$ CI -0.20 to $0.49 ; P=0.41)$; heterogeneity was low $\left(I^{2}=16 \%\right.$; $\left.P=0.28\right)$ (Fig. 3). ${ }^{24,30}$

\section{Quality of life}

Three studies (total $n=179$ ) yielded no significant overall effect $(d=-0.33 ; 95 \%$ CI -0.82 to $0.16 ; P=0.18) .{ }^{25,28,30}$ Heterogeneity was moderate $\left(I^{2}=44 \% ; P=0.17\right)$ (Fig. 4$)$. One study excluded from meta-analysis did not report a significant effect on quality of life. ${ }^{29}$ One intervention was psychotherapy, ${ }^{30}$ and two interventions were system-level. ${ }^{25,28}$

\section{Functioning}

Three studies delivering system-level interventions (total $n=96$ ) yielded no effect $(d=0.02,95 \% \mathrm{CI}-0.38$ to $0.43 ; P=0.91){ }^{16,25,28}$ Heterogeneity between studies was very low $\left(I^{2}=0 \% ; P=0.94\right)$ (Fig. 4).

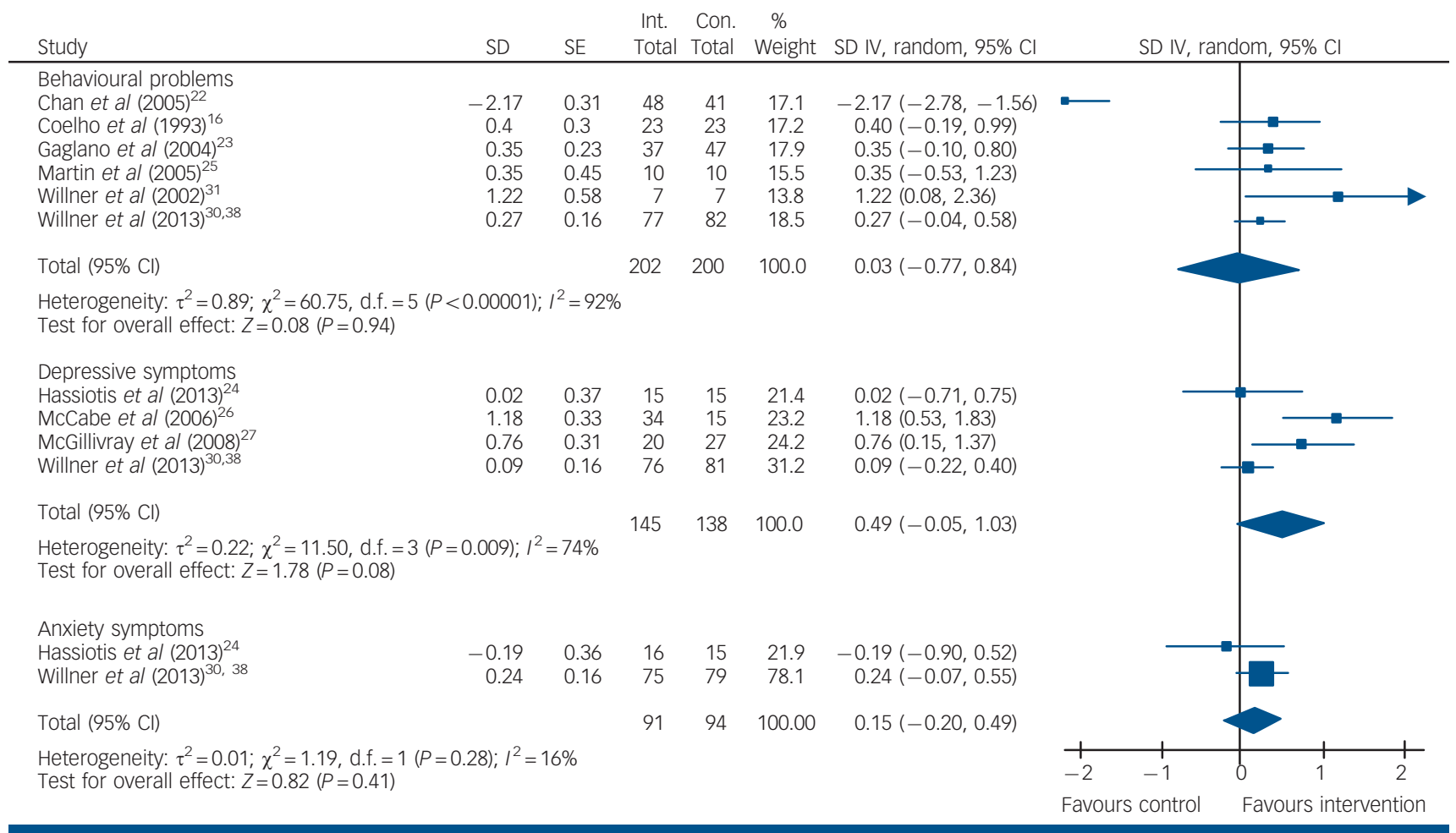

Fig. 3 Forest plot of behavioural problems, depressive and anxiety symptoms. SMD, standardised mean difference; SE, standard error.

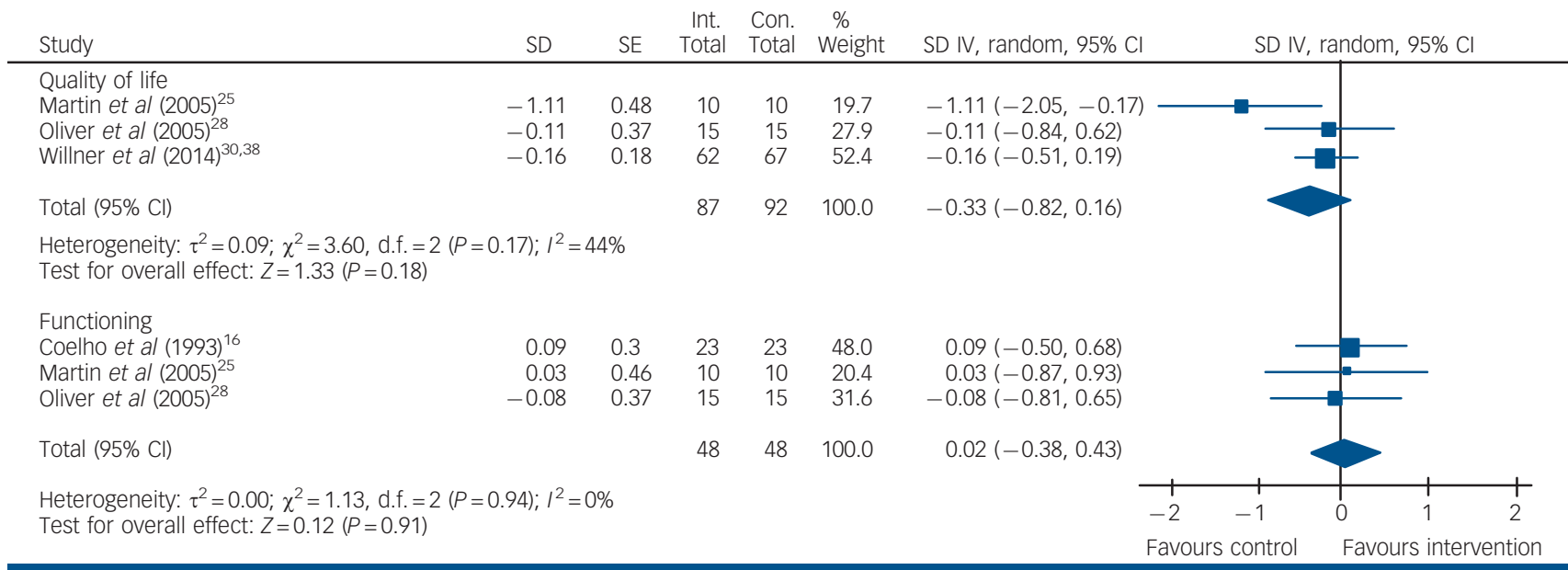

Fig. 4 Forest plot of quality of life and functioning. SMD, standardised mean difference; SE, standard error. 


\section{Discussion}

This systematic review identified 12 controlled studies (total $n=724$ ) which investigated the effectiveness of interventions targeting mental health problems in adults with mild to moderate intellectual disability. Meta-analysis showed that none of a wide range of interventions grouped into psychotherapy, biological and system-level approaches was effective in the sense of yielding statistically significant effect sizes for a number of vital outcome domains including behavioural problems, depression, anxiety, quality of life and functioning.

Our finding for interventions targeting problem behaviours is inconsistent with previous reviews where medium to large effect sizes have been reported for psychosocial therapies, especially $\mathrm{CBT},{ }^{15}$ anger management, ${ }^{12}$ and behavioural interventions. ${ }^{7}$ This might be due to considerable differences in sampling and methods. Vereenooghe \& Langdon included people with all levels of severity of intellectual disabilities, whereas strictly behavioural interventions (e.g. behavioural relaxation), non-traditional and other psychotherapeutic interventions such as life skills training were excluded. ${ }^{15}$ Also, differences from the positive results of a review of 30 controlled studies which also examined the effects of psychological, biological and system or contextual interventions might be due to differences in selection criteria, resulting in fewer studies being analysed. ${ }^{14}$ The review of Hamelin et al was restricted to testing one specific intervention, ${ }^{12}$ and the work of Didden et al was based upon case reports only. ${ }^{7}$ Moreover, only two included studies tested biological interventions, substantiating reservations about using antipsychotics such as risperidone in the treatment of behavioural problems. ${ }^{23}$ Another trial tested 'multisensory therapy', yielding a large negative effect which was a significant outlier. ${ }^{22}$

For the domain of depressive symptoms, the four included trials testing CBT showed a moderate effect size barely below the threshold of statistical significance. This might be a problem of power, i.e. it is possible that only one or two studies more might produce a significant effect. However, it has to be considered that one of the studies showing only a small effect on depression tested CBT focusing on anger rather than on depression. This said, our findings add to the existing evidence for CBT in the treatment of depression in people with mild to moderate intellectual disabilities. $^{6,14,15}$ On the other hand, non-significant negative effects were found for the few interventions targeting quality of life, which were mostly system-level approaches. This finding is in line with another comparable review. ${ }^{13}$ This does not necessarily indicate a general ineffectiveness of system-level interventions, but might point to a lack of distinction between intervention and control conditions where, for example, assertive community treatment might have not added enough to an already strong routine community care. ${ }^{33}$ In addition, the amount of available evidence and sample sizes in the included studies were low. Similarly, interventions targeting the outcome domains anxiety and functioning showed no overall effect.

The methodological quality of the included primary studies varied. Randomisation and masking of participants often were not adequately described, and in ten of the twelve included studies it was not clear whether only selected data had been reported. Moreover, this review showed that the majority of included trials focused on global mental health problems deemed specific to people with intellectual disabilities, such as 'behavioural problems' including poorly defined conditions such as 'aggression' or 'challenging behaviour', rather than focusing on clearly diagnosable mental illnesses such as depression or anxiety, or on outcome domains such as quality of life and functioning for which established standardised measures are available. Also, 'behaviour problems' have usually been assessed using crude, non-standardised staff ratings. Although some behavioural problems may be a manifestation of an underlying mental illness or appear as a comorbid condition to mental illness, ${ }^{34,35}$ it remains a challenge to research to distinguish clearly between mental illness and behavioural problems in people with intellectual disabilities.

\section{Limitations}

Only a small number of studies fulfilled the inclusion criteria, limiting representativeness. Second, heterogeneity of the included studies was substantial, which was especially due to considerable differences in patient characteristics and treatment duration. Third, quite a few of the included studies used waiting lists as a control condition, which might contribute to a 'door-handle' effect, i.e. early and possibly lasting improvement due to the expectation that treatment will start soon. ${ }^{36}$ Fourth, our categorisations of both outcome domains and interventions are open to debate. This was especially obvious for the outcome domain of behavioural problems, which consisted of a mix of poorly defined and measured outcomes. There was also considerable variation in the category of biological interventions.

\section{Implications and outlook}

For the first time the effectiveness of interventions for people with mild to moderate severity of intellectual disabilities and comorbid mental health problems has been systematically reviewed without any restrictions on the type of intervention or outcome domain. These trials represent an important foundation on which further, larger and more rigorous trials can now be designed. At first glance, the main result - there is no compelling evidence supporting interventions aimed at improving mental health problems in people with mild to moderate intellectual disabilities - is rather sobering. Nonetheless, some single interventions showed impressive effects on a number of outcome domains. Future research efforts should focus on the domains of 'genuine' comorbid mental illnesses such as depression and anxiety. Especially CBT seems to be a promising intervention for the treatment of depression, warranting further scrutiny. Finally, efforts should be made toward disentangling the commonly used and nevertheless questionable outcome 'behavioural problems', primarily aiming at the development of a sound definition and adequate measurement.

\footnotetext{
Nadine Koslowski, MA, Kristina Klein, MA, Katrin Arnold, Dipl-Soc, Markus Kösters, PhD, Department of Psychiatry II, UIm University; Matthias Schützwohl PhD, Department of Psychiatry and Psychotherapy, University Hospital Carl Gustav PhD, Department of Psychiatry and Psychotherapy, University Hospital Carl Gustav
Carus, Technische Universität Dresden; Hans Joachim Salize, PhD, Mental Health Services Research Group, Central Institute of Mental Health, Mannheim; Bernd Puschner, PhD, Department of Psychiatry II, UIm University, Germany

Correspondence: Dr Bernd Puschner, Section Process-Outcome Research, Department of Psychiatry II, UIm University, Ludwig-Heilmeyer-Str. 2, D-89312 Günzburg, Germany. Email: bernd.puschner@bkh-guenzburg.de

First received 8 Oct 2014, final revision 29 May 2015, accepted 1 Aug 2015
}

\section{Funding}

This work is part of the study Mental Health Care Provision for Adults with Mental Retardation and a Psychiatric Diagnosis (MEMENTA) which has been funded by the German Federal Ministry for Education and Research (grant number 01GY1134).

\section{Acknowledgements}

We are grateful to Professor Thomas Becker, Department of Psychiatry II, UIm University, Germany, for useful comments on an earlier manuscript version. 


\section{References}

1 Dosen A. Diagnosis and treatment of psychiatric and behavioural disorders in mentally retarded individuals: the state of the art. J Intellect Disabil Res 1993; 37: 1-7.

2 Borthwick-Duffy SA. Epidemiology and prevalence of psychopathology in people with mental retardation. J Consult Clin Psychol 1994; 62: 17-27.

3 Deb S, Thomas M, Bright C. Mental disorder in adults with intellectual disability. 1: prevalence of functional psychiatric illness among a community based population aged between 16 and 64 years. J Intellect Disabil Res 2001; 45: 495-505.

4 Cooper S-A, Smiley E, Morrison J, Williamson A, Allan L. Mental ill-health in adults with intellectual disabilities: prevalence and associated factors. Br J Psychiatry 2007; 190: 27-35.

5 Brown JF, Brown MZ, Dibiasio P. Treating individuals with intellectual disabilities and challenging behaviors with adapted dialectical behavio therapy. J Ment Health Res Intellect Disabil 2013; 6: 280-303.

6 Hatton C. Psychosocial interventions for adults with intellectual disabilities and mental health problems: a review. J Ment Health 2002; 11: 357-74.

7 Didden R, Korzilius $\mathrm{H}$, van Oorsouw W, Sturmey P. Behavioral treatment of challenging behaviors in individuals with mild mental retardation: meta-analysis of single-subject research. Am J Ment Retard 2006; 111: 290-8.

8 Chan JS, Tse SH. Massage as therapy for persons with intellectual disabilities: a review of the literature. J Intellect Disabil 2011; 15: 47-62.

9 Ulzen TP, Powers RE. A review of empirical evidence of somatic treatment options for the MI/DD population. Psychiatr Q 2008; 79: 265-73.

10 Deb S, Sohanpal SK, Soni R, Lenotre L, Unwin G. The effectiveness of antipsychotic medication in the management of behaviour problems in adults with intellectual disabilities. J Intellect Disabil Res 2007; 51: 766-77.

11 Duggan L, Brylewski J. Antipsychotic medication versus placebo for people with both schizophrenia and learning disability. Cochrane Database Syst Rev 2004; 4: CD000030.

12 Hamelin J, Travis R, Sturmey P. Anger management and intellectual disabilities: a systematic review. J Ment Health Res Intellect Disabil 2013; 6: $60-70$.

13 Balogh $\mathrm{R}$, Ouellette-Kuntz $\mathrm{H}$, Bourne $\mathrm{L}$, Lunsky $\mathrm{Y}$, Colantonio A Organising health care services for persons with an intellectual disability. Cochrane Database Syst Rev 2008; 8: CD007492.

14 Heyvaert M, Maes B, Onghena P. A meta-analysis of intervention effects on challenging behaviour among persons with intellectual disabilities. J Intellect Disabil Res 2010; 54: 634-49.

15 Vereenooghe L, Langdon PE. Psychological therapies for people with intellectual disabilities: a systematic review and meta-analysis. Res Dev Disabil 2013; 34: 4085-102.

16 Coelho RJ, Kelley PS, Deatsman-Kelly C. An experimental investigation of an innovative community treatment model for persons with a dual diagnosis (DD/MI). J Rehabil 1993; 59: 37-42.

17 Moher D, Liberati A, Tetzlaff J, Altman DG. Preferred reporting items for systematic reviews and meta-analyses: the PRISMA statement. PLOS Med 2009; 6: e1000097.

18 Dunn LM, Dunn L, Whetton C, Pintilie D. British Picture Vocabulary Scale. nfer-Nelson, 1982

19 Beech JR, Keys A. Reading, vocabulary and language preference in 7- to 8-year-old bilingual Asian children. Br J Educ Psychol 1997; 67: 405-14.

20 Higgins JPT, Green S (eds). Cochrane Handbook for Systematic Reviews of Interventions, 5th edn. Wiley-Blackwell, 2011.

21 Cohen J. Statistical Power Analysis for the Behavioral Sciences, 2nd edn. Erlbaum, 1988.
22 Chan S, Fung MY, Tong CW, Thompson D. The clinical effectiveness of a multisensory therapy on clients with developmental disability. Res Dev Disabil 2005; 26: 131-42.

23 Gagiano C, Read S, Thorpe L, Eerdekens M, van Hove I. Short- and long-term efficacy and safety of risperidone in adults with disruptive behavior disorders. Psychopharmacology 2005; 179: 629-36.

24 Hassiotis A, Serfaty M, Azam K, Strydom A, Blizard R, Romeo R, et al. Manualised individual cognitive behavioural therapy for mood disorders in people with mild to moderate intellectual disability: a feasibility randomised controlled trial. J Affect Disord 2013; 151: 186-95.

25 Martin G, Costello $H$, Leese $M$, Slade $M$, Bouras $N$, Higgins $S$, et al. An exploratory study of assertive community treatment for people with intellectual disability and psychiatric disorders: conceptual, clinical, and service issues. J Intellect Disabil Res 2005; 49: 516-24.

26 McCabe MP, McGillivray JA, Newton DC. Effectiveness of treatment programmes for depression among adults with mild/moderate intellectual disability. J Intellect Disabil Res 2006; 50: 239-47.

27 McGillivray JA, McCabe MP, Kershaw MM. Depression in people with intellectual disability: an evaluation of a staff-administered treatment program. Res Dev Disabil 2008; 29: 524-36.

28 Oliver PC, Piachaud J, Tyrer P, Regan A, Dack M, Alexander R, et al. Randomized controlled trial of assertive community treatment in intellectual disability: the TACTILD study. J Intellect Disabil Res 2005; 49: 507-15.

29 Tyrer P, Oliver-Africano PC, Ahmed Z, Bouras N, Cooray S, Deb S, et al. Risperidone, haloperidol, and placebo in the treatment of aggressive challenging behaviour in patients with intellectual disability: a randomised controlled trial. Lancet 2008; 371: 57-63.

30 Willner $\mathrm{P}$, Rose J, Jahoda A, Stenfert Kroese B, Felce D, Cohen D, et al. Group-based cognitive-behavioural anger management for people with mild to moderate intellectual disabilities: cluster randomised controlled trial. Br J Psychiatry 2013; 203: 288-96.

31 Willner $\mathrm{P}$, Jones J, Tams R, Green G. A randomized controlled trial of the efficacy of a cognitive-behavioural anger management group for clients with learning disabilities. J Appl Res Int Dis 2002; 15: 224-35.

32 Benson BA, Rice CJ, Miranti SV. Effects of anger management training with mentally retarded adults in group treatment. J Consult Clin Psychol 1986; 54 728-9.

33 Burns T. End of the road for treatment-as-usual studies? Br J Psychiatry 2009; 195: 5-6

34 Allen D, Davies D. Challenging behaviour and psychiatric disorder in intellectual disability. Curr Opin Psychiatry 2007; 20: 450-5.

35 Deb S, Chaplin R, Sohanpal S, Unwin G, Soni R, Lenotre L. The effectiveness of mood stabilizers and antiepileptic medication for the management of behaviour problems in adults with intellectual disability: a systematic review. J Intellect Disabil Res 2008; 52: 107-13.

36 Gillespie R. Manufacturing Knowledge: A History of the Hawthorne Experiments. CUP, 1993

37 Tyrer $\mathrm{P}$, Oliver-Africano $\mathrm{P}$, Romeo R, Knapp $\mathrm{M}$, Dickens $\mathrm{S}$, Bouras $\mathrm{N}$, et al. Neuroleptics in the treatment of aggressive challenging behaviour for people with intellectual disabilities: a randomised controlled trial (NACHBID). Health Technol Assess 2009; 13: 1-54.

38 Willner $\mathrm{P}$, Jahoda A, Rose J, Stenfert Kroese B, Hood K, Townson JK, et al. Anger management for people with mild to moderate learning disabilities: study protocol for a multi-centre cluster randomized controlled trial of a manualized intervention delivered by day-service staff. Trials 2011; 12: 36 .

39 Willner $\mathrm{P}$, Rose J, Jahoda A, Stenfert Kroese B, Felce D, MacMahon P, et al. A cluster randomised controlled trial of a manualised cognitive behavioural anger management intervention delivered by supervised lay therapists to people with intellectual disabilities. Health Technol Assess 2013; 17: 1-173. 